\title{
Correction to: Isolation and Characterization of a microRNA-size Secretable Small RNA in Streptococcus sanguinis
}

\author{
Ji-Woong Choi ${ }^{1} \cdot$ Tae-Yub Kwon $^{2} \cdot$ Su-Hyung Hong ${ }^{1} \cdot$ Heon-Jin Lee ${ }^{1}{ }^{1}$
}

Published online: 20 March 2018

(c) Springer Science+Business Media, LLC, part of Springer Nature 2018

\section{Correction to: Cell Biochem Biophys}

https://doi.org/10.1007/s12013-016-0770-5;

Published online 31 October 2016.

The original version of this article unfortunately contains a mistake in the fund number under Acknowledgment section. The funding number should read as "2017R1A5A2015391 and 2015R1D1A3A01019684".

The original article can be found online at https://doi.org/10.1007/ s12013-016-0770-5.

Heon-Jin Lee

heonlee@knu.ac.kr

1 Department of Oral Microbiology and Immunology, School of Dentistry, Kyungpook National University, Daegu, Korea

2 Department of Dental Biomaterials, School of Dentistry, Kyungpook National University, Daegu, Korea 